\title{
AN ANALYSIS OF JARGON USED IN POLITICAL WEBSITE
}

\author{
Dewi Syafitri ${ }^{1}$ \\ STKIP PGRI LUBUKLINGGAU \\ M. Ikhsan ${ }^{2}$ \\ STKIP PGRI LUBUKLINGGAU \\ syafitri2019@gmail.com ${ }^{1}$
}

Submit, 20-05-2019 Accepted, 21-06-2019 Publish, 23-06-2019

\begin{abstract}
The objectives of the study were to describe the forms, meanings, and functions of Political Jargon Which were found in Political Website. This study mainly aimed to describe the jargon used in political news of CNN politics as the Political Website. The writers used a qualitative descriptive method in classifying and analyzing the data. After investigating the forms, meanings, and functions of jargon in CNN politics on $1^{\text {st }}-30^{\text {th }}$ May, 2016, the writers found several findings. The political jargon in this website can be analyzed in forms were 53 words, 15 phrases, 5 abbreviations and there was no acronym, in the lexical meanings categories there were 58 denotative meanings and 15 connotative meanings from 73 data and the functions of Political Jargon were a belongings to a specific group, to communicate and signal for identification of political group. In conclusion, the jargon used to convey the meaning and to communicate to the readers, in this context as a signal in politics fields. So, the readers will be familiar in using some terms of political jargon in everyday speech.
\end{abstract}

Keywords: jargon, political website, meaning

\section{INTRODUCTION}

Jargon is one of the language variations in sociolinguistics. Jargon was the special language that used in the certain field in the society. According to Jendra (2010), jargon is words with special meanings related to the professional's fields. There are many words that do not understand by the society in general and the outside of the group. Jargon has many functions, it can identification the people activity and it can be easier in communication with others in some field but for people that do not know these words was not interest to follow in the conversation because they do not understood. Therefore the language in jargon was different in common language and people in the outside of the group difficult to understand this word.

Jargon was usually found in the professional fields specifically in politics. In this study, the writers focused on CNN Political Website as source of the data. 
The CNN Website was one of some international website which published in internet. It might be one of the English-language media that still exist until now. According to Ipsos (2014:34), in that the latest IPSOS Affluent Global Survey reaffirms $\mathrm{CNN}$ as the leading international news brand in Europe, Middle East, Africa, Asia and Latin America, and from the IPSOS Affluent Survey Asia pacific, shows every mount CNN is consumed $34 \%$ of region's affluent audience which is more than the largest news brand (BBC World News only 24\%) and (CNBC only $16 \%$ ).

The CNN website captivates the reader with a variety of cultures, there were many news pages such as political, healthy, opinion, archipelago, business, world, special issues, etc. According to Drezner and Farrel (2008:16) on alex.com survey which accessed 20 January $2005 \mathrm{CNN}$ was $26^{\text {th }}$ rank of $10285^{\text {th }}$ rank in alexa ranking and $8^{\text {th }}$ rank of $10^{\text {th }}$ rank in page rank google and 20.030 reach per million user.

According to Satyakumara (2013:23), CNN is the $2^{\text {nd }}$ rank of 15 top popular news website in Indonesia. Jargon is a special language belongs to exclusively to a group, often a profession. Engineers, lawyers, doctors, tax analysts, sport players, and the like all use jargon to exchange complex information efficiently. Every field in this life used the jargon to make easy in communication. Political website was one of the fields that use jargon. Nowadays, many people like to read the political news by using the website that from teenager and adult.

Jargon in Political Website was familiar in our life and many people using it. The meaning of utterance of the Political Website used cannot be known and understood by user. The use of jargon in Political Website was very advantageous because it will easier in communication with others. Using jargon was the effective ways to communicate between the groups in the some profession. Therefore the writers were interested in analyzing jargon use of political website. According to Drezner and Farrel (2008:15), the website had far less reach than other online media outlets for political information and analysis- talk radio, cable news channels, or other online political websites.

Based on the explanation above, the writers were interested analyzing the form, meaning and function of Jargon in political website, specifically in The CNN Political Website. In this website, the writers found frequently usage of political language that encountered in political news pages. It also created a significant portion in reading and writing of gadget user, especially the young generation shaves to know about the global situation in the politics. When the reader understand meaning, form, and function of jargon politics would easier to analyze some blogs, news pages, articles, journals, novels, books, and etc. Besides, jargon used in political website giving the benefit to improve the 
vocabulary. The data were selected from the news pages which were published on $1^{\text {st }}-30^{\text {th }}$, May 2016. This research served the common used of political jargon and also the meaning with various vocabulary found in international political website.

\section{LITERATURE REVIEW}

\section{The Concept of Jargon}

Jargon is varieties of language created for specific functions by the people who engage in them regularly. It is like mini dialects but used only for the activity for which they were created. Other definition about jargon is more specifically stated in Oxford Advanced Learner's Dictionary (2001: 693), states that jargon is technical words expression used by a particular profession or group of people and difficult for others to understand: medical/legal/computer jargon. So, people in certain groups or communities use jargon on many purposes.

Jargon usually means the special language of a group that people outside the group do not understand. Trades and profession have specialized terms that people who work in the field know. When the people are writing for general a general audience however avoids jargon and use terms that everyone understand. According to Jendra (2010:58) the language used among members of a professional field is usually different from the one use in other fields.

Jargon use as the label for vocabulary peculiar to some field or occupational. Other group or communities who want to know the language should learn and interact with those communities. Thus, in English for instance, the word such us epidemics, prescription, contagion are found to be used by the medical professionals. Whereas, big government, bipartisan, campaign are used in politics. According to Jendra (2010:59) specialty words or jargons may be used by professional for different purposes.

The language often cannot be understood in the general society or the society in the society in the outside of the group. For example in the jargon of computer likes mouse, keyboard, RAM. The people know about these words but still confuse to interpret the meaning because jargon consists of technical words used by certain people in a certain social or professional group.

Sometimes the same words can indeed express different meanings when they are used in different fields. The word "operation" for instance, means 'soldier's attack to a targeted area of war in military, but in the medical domains the word is used to mean 'a certain act by hospital surgeons of helping a sick person'. The words 'private' is commonly used in the commercial fields to mean 'not belong to public or government' such as in 'privet' company, 'privet' business, etc. 
Jargon used to communicate and interact between the people in one group in the society. According to Yuniasih (2013:11-14) jargon has many forms like in word, meanings, and functions.

\section{Forms of Jargon}

\section{Word}

According to Kridalaksana (2008:101), word is the smallest unit of language that the people can understand if it is said or written on its own. For example, the word of jargon forms in computer such as hardware (the physical parts of a computer) and virus (a program that has been deliberately created to cause computer problems).

\section{Phrase}

According to Kridalaksana (2008:66), phrase is two or more words that work together as a unit. The example of jargon in the phrase form such as in economic likes delivery order (a document from the owner or holder of the goods requesting the release of goods held under warrant).

\section{Abbreviation}

According to Kridalaksana (2008:1), abbreviation is the result of shortened unit. The way of shortened takes the first syllable from the words. For example of jargon in the abbreviation form in the electronic MMS (Multimedia Messaging Service).

\section{Acronym}

According to Krisdalaksana (2008:5), in the other hand, acronym is the word. The meaning of the word is the long of that word. The example of jargon in the acronym from computer is like modem (Modulator/Demodulator, a device for allowing computers to communicate over a phone line).

\section{The Meanings of Jargon}

Meaning is what is referred or indicated by e. g. sounds, words, or signals. Meaning can be divided into two elements like lexical and grammatical meaning. Lexical meaning is the meaning given to linguistics element. Grammatical meaning is meaning brought the pattern in the language. Meaning is the way or how the people utter the words so the hearer understands what the people speak or write. According to Boer (1973:42-43), there are two kinds of words as followings:

\section{Denotative Meaning}

Denotative is the natural meaning and no addition. The meaning in the word not connected with the other thing, not explain in the connection with the 
other event. The denotative meaning is the meaning that suitable with the definition in the dictionary and it is limited. For example medicine has the denotative meaning the art and science of the prevention and cure of disease.

\section{Connotative Meaning}

The connotative meaning must be learnt and mastered based on the language experience and the usage. Some of the connotative meaning has been write on the dictionary if these meaning become to general connotation. It is the subjective because there are many additional feelings and values. If the denotative meaning can be understood by many people, the connotative meaning only can be understood by the small number of people. The connotative meaning is depend on the personal interpretation. For example, itchy has the connotative meaning having or producing irritation on the skin.

\section{The Functions of Jargon}

Jargon is a specialized term that has special function in communication. Each profession needs jargon. Based on Ives in Yuniasih (2013:18) jargon has some function as follows: 1) jargon can give a person a sense of belonging to a specific group; 2) jargon can also make it easier for a person to communicate with their friend, 3) jargon is effective as signals for identification.

Table 1 Political Jargon Classification

\begin{tabular}{llll}
\hline $\begin{array}{c}\text { Political } \\
\text { jargon } \\
\text { (example) }\end{array}$ & $\begin{array}{c}\text { Forms of } \\
\text { jargon }\end{array}$ & \multicolumn{1}{c}{ Meaning of jargon } & \multicolumn{1}{c}{$\begin{array}{c}\text { Function of } \\
\text { jargon }\end{array}$} \\
\hline Left & Word & $\begin{array}{l}\text { On the liberal side of politic spectrum } \\
\text { (denotative) }\end{array}$ & $\begin{array}{l}\text { For a person to } \\
\text { communicate }\end{array}$ \\
\hline $\begin{array}{l}\text { Blue dog } \\
\text { democrat }\end{array}$ & Phrase & $\begin{array}{l}\text { Coined in the mid-1980s for a conservative } \\
\text { Southern democrat, inspired by yellow dog } \\
\text { democrat and the paintings of Gorge } \\
\text { of belonging to a } \\
\text { Rodrigue (connotative ) }\end{array}$ \\
\hline GOP & Abbreviation & $\begin{array}{l}\text { Grand Old Party nickname of the } \\
\text { Republican party (connotative ) }\end{array}$ & $\begin{array}{l}\text { Signal fon } \\
\text { identification }\end{array}$ \\
\hline Photo-Op & Acronym & $\begin{array}{l}\text { Short for "photo opportunity" an event } \\
\text { staged specially for news cameras to help a } \\
\text { politician appear on the evening news in } \\
\text { morning papers (connotative) }\end{array}$ \\
\hline
\end{tabular}

http://www.scholastic.com/teachers/article/vocabulary-political-words

\section{The Concept of Political Website}

Website is an information page which provided by using the internet so it can be access in a global area during it is connected with the internet connection. Website is the component or group of component that consist of texts, pictures, audios, videos, animation, etc. (Bowo, 2014:4). A website can be access by using 
a computer, smart mobile or the other gadget in this era. Usually the information in the website is saved in the webserver which is managed by a person or an institution.

Politics is one of the famous topic in the website for the society because in their life they are very close with the politics and government. So important to know the language use in website politic which gives the all information about the political action in the word, several of website politics use the politics language or jargon politics for the website use. According to Markus in Jogiyanto (2009:623624), a system of information or website will give the influence for the power of politic and organization for the reason as follows: 1) the access of information will influence the value of a judgment; 2) the system of information use for allocation of sources information will influence the personality of the people, 3) the system of information use for control and prohibit actions, 4) the system information cause a dominance and a power because it have a capability to changes the result.

\section{RESEARCH METHOD}

This study useddescriptive qualitative research because the data were described and analyzed in words. The qualitative method was a method which focused on words matter than numbers and there was no statistical procedure.

This study discussed or analyzed of jargon used in the Political Website in the news page of CNN. The writers used the news page of CNN to found out the forms of Politic Jargon. After the data were collected they classified into forms categories consisting:words, phrase, abbreviation, and acronym. Then the writersclassified lexical meaning of jargon categories consisting: denotative and connotative meaning. And the writers made description about function of jargon used based on content of the news page.

Technique for collecting the data was a technique to get and collect the data. To obtainthe data, the writersused the Political Website/CNN.com as the data source and focused on the Politics jargon used. The data took on the news pages in the CNN.com politics news pagesthat published on May $1^{\text {st }}-30^{\text {th }}, 2016$. Then, the data analyzed in several steps including identification, classification and description.

\section{FINDING}

In this study the writers used 30 titles of news pages about politics and american legislative in $\mathrm{CNN}$ politics.

\section{The Forms of Political Jargon in Political Website}

There are four classifications of Jargon based on the forms of words groups, namely in single words, phrase, abbreviation and acronym. The words were indentified from the CNN politics on $1^{\text {st }}-30^{\text {th }}$, May 2016 as follows: 


\section{Word Form}

The following table presents the word form of jargon in Political Website:

Table 2 The Words Form of Jargon in Political Website

\begin{tabular}{|c|c|c|c|c|}
\hline No & Words Form & $\begin{array}{c}\text { Meaning of Jargon in Political } \\
\text { Website }\end{array}$ & $\begin{array}{c}\text { Codes title of news } \\
\text { pages in CNN } \\
\text { Politics } \\
\end{array}$ & $\begin{array}{l}\text { Word } \\
\text { classes }\end{array}$ \\
\hline 1. & Accountability & $\begin{array}{l}\text { The fact or condition of being } \\
\text { accountable }\end{array}$ & $\mathrm{CNN} / 5 / 8^{\text {th }} / 16$ & Noun \\
\hline 2. & Addressed & $\begin{array}{l}\text { To provide words on a topic } \\
\text { which may have little or no } \\
\text { meaning }\end{array}$ & $\mathrm{CNN} / 5 / 10^{\text {th }} / 16$ & Noun \\
\hline 3. & Ballot & $\begin{array}{l}\text { A system of voting secretly and } \\
\text { writing on a particular issue / the } \\
\text { piece of paper used to record a } \\
\text { person's vote. }\end{array}$ & $\mathrm{CNN} / 5 / 3^{\mathrm{rd}}$ and $12^{\mathrm{th}} / 16$ & Noun \\
\hline 4. & Blowback & $\begin{array}{l}\text { The unintended adverse result of } \\
\text { a political action or situation. }\end{array}$ & $\mathrm{CNN} / 5 / 10^{\mathrm{th}} / 16$ & Noun \\
\hline 5. & Blowout & $\begin{array}{l}\text { An easy victory in a supporting } \\
\text { contest or election. }\end{array}$ & $\begin{array}{l}\mathrm{CNN} / 5 / 17^{\mathrm{th}} \text { and } \\
18^{\mathrm{th}} / 16\end{array}$ & Noun \\
\hline 6. & Budget & $\begin{array}{l}\text { Projected dollar amounts } \\
\text { estimated ahead of time for a } \\
\text { project. }\end{array}$ & $\mathrm{CNN} / 5 / 12^{\text {th }} / 16$ & Noun \\
\hline 7. & Bombshell & $\begin{array}{l}\text { An unexpected and surprising } \\
\text { event, especially an unpleasant } \\
\text { one. }\end{array}$ & $\mathrm{CNN} / 5 / 13^{\text {th }} / 16$ & Noun \\
\hline & Caucus & $\begin{array}{l}\text { An information meeting of local } \\
\text { party members to discuss } \\
\text { candidates and choose delegates } \\
\text { to the party convention. }\end{array}$ & $\mathrm{CNN} / 5 / 2^{\text {nd }}$ and $13^{\text {th }} / 16$ & Noun \\
\hline & Confident & $\begin{array}{l}\text { Assurance that a plan of action } \\
\text { will work out to imply prior } \\
\text { knowledge of future events }\end{array}$ & $\begin{array}{l}\mathrm{CNN} / 5 / 6^{\text {th }}, 9^{\text {th }}, 10^{\text {th }}, \\
\text { and } 11^{\text {th }} / 16\end{array}$ & $\begin{array}{l}\text { Adjectiv } \\
\text { e }\end{array}$ \\
\hline 10. & Campaigner & $\begin{array}{l}\text { A person who work in an } \\
\text { organized and active way towards } \\
\text { a goal. }\end{array}$ & $\mathrm{CNN} / 5 / 22^{\text {nd }} / 16$ & Noun \\
\hline 11. & Candidate & $\begin{array}{l}\text { A person who applies for a job } \\
\text { nominated for election. }\end{array}$ & $\begin{array}{l}\text { CNN } / 5 / 2^{\text {nd }}, 4^{\text {th }}, 7^{\text {th }}, \\
8^{\text {th }}, 10^{\text {th }}, 11^{\text {th }}, 12^{\text {th }}, \\
13^{\text {th }}, 16^{\text {th }}, 19^{\text {th }}, 20^{\text {th }}, \\
22^{\text {nd }}, 25^{\text {th }}, 26^{\text {th }} / 16\end{array}$ & Noun \\
\hline 12. & Campaign & $\begin{array}{l}\text { An organized effort to win an } \\
\text { election }\end{array}$ & $\begin{array}{l}\mathrm{CNN} / 5 / 2^{\text {nd }}, 4^{\text {th }}, 7^{\text {th }}, \\
8^{\text {th }}, 10^{\text {th }}, 11^{\text {th }}, 12^{\text {th }}, \\
13^{\text {th }}, 16^{\text {th }}, 19^{\text {th }}, 20^{\text {th }}, \\
22^{\text {nd }}, 25^{\text {th }}, 26^{\text {th }} / 16\end{array}$ & Verb \\
\hline
\end{tabular}




\begin{tabular}{|c|c|c|c|}
\hline 13. Chairwoman & A female chairperson. & $\mathrm{CNN} / 5 / 29^{\mathrm{th}} / 16$ & Noun \\
\hline 14. Coalition & $\begin{array}{l}\text { A temporary alliance for } \\
\text { combined action, especially of } \\
\text { political parties forming a } \\
\text { government. }\end{array}$ & $\mathrm{CNN} / 5 / 3^{\text {rd }} / 16$ & Noun \\
\hline 15. Democratic & $\begin{array}{l}\text { Relating or supporting } \\
\text { democracy or its principles }\end{array}$ & $\begin{array}{l}\mathrm{CNN} / 5 / 1^{\mathrm{st}}, 3^{\text {rd }}, 6^{\text {th }}, \\
8^{\text {th }}, 9^{\text {th }} / 16,10^{\text {th }}, 11^{\text {th }}, \\
18^{\text {th }}, 22^{\text {nd }}, 23^{\text {rd }}, 24^{\text {th }}, \\
29^{\text {th }}, 30^{\text {th }} / 16\end{array}$ & $\begin{array}{l}\text { Adjectiv } \\
\mathrm{e}\end{array}$ \\
\hline 16. Democracy & $\begin{array}{l}\text { A system of government by the } \\
\text { whole population or all the } \\
\text { eligible members of a state, } \\
\text { typically through elected } \\
\text { representative }\end{array}$ & $\begin{array}{ll}\mathrm{CNN} / 5 / 4^{\text {th }} & \text { and } \\
12^{\text {th }} / 16 & \end{array}$ & Noun \\
\hline 17. Democrat & $\begin{array}{l}\text { A member of the Democratic } \\
\text { Party. }\end{array}$ & $\begin{array}{l}\mathrm{CNN} / 5 / 5^{\text {th }}, 6^{\text {th }}, 9^{\text {th }}, \\
10^{\text {th }}, 20^{\text {th }}, 22^{\text {nd }}, 23^{\text {rd }}, \\
24^{\text {th }} / 16\end{array}$ & Noun \\
\hline 18 Debate & $\begin{array}{l}\text { Held between the democratic } \\
\text { nominees, republican nominees, } \\
\text { as well as in the presidential } \\
\text { campaign. }\end{array}$ & $\begin{array}{l}\mathrm{CNN} / 5 / 4^{\text {th }} \quad \text { and } \\
25^{\text {th }} / 16\end{array}$ & Noun \\
\hline 19. Delegate & $\begin{array}{l}\text { A person chosen at local level to } \\
\text { represent the state at the part's } \\
\text { National Convention. }\end{array}$ & $\begin{array}{l}\mathrm{CNN} / 5 / 2^{\text {nd }}, 3^{\text {rd }}, 6^{\text {th }}, \\
10^{\text {th }}, 11^{\text {th }}, 23^{\text {rd }}, 29^{\text {th }}, \\
30^{\text {th }} / 16\end{array}$ & Noun \\
\hline 20. Declaration & $\begin{array}{l}\text { A formal or explicit statement or } \\
\text { announcement. }\end{array}$ & $\mathrm{CNN} / 5 / 8^{\text {th }} / 16$ & Noun \\
\hline 21. Election & $\begin{array}{l}\text { A formal and organized choice by } \\
\text { vote of a person for political } \\
\text { officer or other position }\end{array}$ & $\begin{array}{l}\mathrm{CNN} / 5 / 1^{\mathrm{st}}, 3^{\mathrm{rd}}, 6^{\text {th }}, \\
7^{\text {th }}, 8^{\text {th }}, 10^{\text {th }}, 15^{\text {th }}, 12^{\text {th }}, \\
23^{\text {rd }}, 13^{\text {th }}, 14^{\text {th }}, \\
17^{\text {th }} / 16\end{array}$ & Noun \\
\hline 22. Endorsement & $\begin{array}{l}\text { Come in three types: from } \\
\text { celebrities, newspaper, and } \\
\text { political figures and organization. }\end{array}$ & $\mathrm{CNN} / 5 / 20^{\text {th }} / 16$ & Noun \\
\hline 23. Endorse & $\begin{array}{l}\text { Declare one's public approval or } \\
\text { support of. }\end{array}$ & $\begin{array}{l}\mathrm{CNN} / 5 / 6^{\text {th }}, 10^{\text {th }}, 11^{\text {th }}, \\
12^{\text {th }}, 13^{\text {th }}, 23^{\text {rd }} / 16\end{array}$ & Verb \\
\hline 24. Escaping & $\begin{array}{l}\text { Break free from confinement or } \\
\text { control }\end{array}$ & $\mathrm{CNN} / 5 / 8^{\text {th }} / 16$ & Verb \\
\hline 25. Establishment & $\begin{array}{l}\text { A group in a society exercising } \\
\text { power and influence over matters } \\
\text { of policy, opinion, or taste and } \\
\text { seen as resisting change. }\end{array}$ & $\begin{array}{l}\mathrm{CNN} / 5 / 6^{\text {th }}, \\
24^{\text {th }} / 16\end{array}$ & Noun \\
\hline 26. Electorate & $\begin{array}{l}\text { All the people in a country or area } \\
\text { who are entitled to vote in an } \\
\text { election. }\end{array}$ & $\mathrm{CNN} / 5 / 23^{\mathrm{rd}} / 26$ & Noun \\
\hline
\end{tabular}




\begin{tabular}{|c|c|c|c|}
\hline 27. House & $\begin{array}{l}\text { A legislative or declarative } \\
\text { assembly }\end{array}$ & $\begin{array}{l}\mathrm{CNN} / 5 / 3^{\text {rd }}, 7^{\text {th }}, 12^{\text {th }}, \\
13^{\text {th }}, 23^{\text {rd }}, 26^{\text {th }} / 16\end{array}$ & Noun \\
\hline 28. Ideological & $\begin{array}{l}\text { Based on or relating to a system } \\
\text { of ideas and ideals, especially } \\
\text { concerning economic or political } \\
\text { theory and policy. }\end{array}$ & $\mathrm{CNN} / 5 / 3^{\text {rd }}$ and $7^{\text {th }} / 16$ & $\begin{array}{l}\text { Adjectiv } \\
\mathrm{e}\end{array}$ \\
\hline 29. Ideology & $\begin{array}{l}\text { A system of ideas and ideals, } \\
\text { especially one that forms the } \\
\text { basic of economy or political } \\
\text { theory and policy }\end{array}$ & $\begin{array}{l}\mathrm{CNN} / 5 / 6^{\text {th }} \text { and } 26^{\text {th }} \\
/ 16\end{array}$ & Noun \\
\hline 30. Leftist & $\begin{array}{l}\text { A person who supports the } \\
\text { political views or policies of left. }\end{array}$ & $\mathrm{CNN} / 5 / 5^{\text {th }} / 16$ & Noun \\
\hline 31. Liberal & $\begin{array}{l}\text { Willing to respect or accept } \\
\text { behavior or opinions different } \\
\text { from one's own: open to new } \\
\text { idea. }\end{array}$ & $\begin{array}{l}\mathrm{CNN} / 5 / 5^{\text {th }}, 7^{\text {th }}, 24^{\text {th }}, \\
26^{\text {th }} / 16\end{array}$ & $\begin{array}{l}\text { Adjectiv } \\
\text { e }\end{array}$ \\
\hline 32. Left & $\begin{array}{l}\text { Relating to a person or group } \\
\text { favouring radical, reforming, or } \\
\text { socialist views. }\end{array}$ & $\mathrm{CNN} / 5 / 5^{\text {th }} / 16$ & $\begin{array}{l}\text { Adjectiv } \\
\text { e }\end{array}$ \\
\hline Nomination & $\begin{array}{l}\text { The action of political party } \\
\text { choose is official candidate for a } \\
\text { particular office. }\end{array}$ & $\begin{array}{l}\mathrm{CNN} / 5 / 1^{\mathrm{st}}, 2^{\text {nd }}, 3^{\text {rd }} \\
4^{\text {th }}, 5^{\text {th }}, 6^{\text {th }}, 11^{\text {th }}, 13^{\text {th }} \\
/ 16\end{array}$ & Noun \\
\hline 34. Nominee & $\begin{array}{l}\text { The candidate chosen by a } \\
\text { political party to run for a } \\
\text { particular office. }\end{array}$ & $\begin{array}{l}\mathrm{CNN} / 5 / 2^{\text {nd }}, 3^{\text {rd }}, 4^{\text {th }}, \\
5^{\text {th }}, 6^{\text {th }}, 7^{\text {th }}, 9^{\text {th }}, 10^{\text {th }}, \\
11^{\text {th }}, 12^{\text {th }}, 13^{\text {th }}, 25^{\text {th }}, \\
26^{\text {th }}, 28^{\text {th }} / 16\end{array}$ & Noun \\
\hline 35. Opposition & $\begin{array}{l}\text { A group of opponents / resistance } \\
\text { or dissent, expressed in action or } \\
\text { argument. }\end{array}$ & $\mathrm{CNN} / 5 / 3^{\mathrm{rd}}$ and $7^{\text {th }} / 16$ & Noun \\
\hline 36. Opportunity & $\begin{array}{l}\text { A problem which does not have a } \\
\text { clear solution Someone's } \\
\text { assignment }\end{array}$ & $\begin{array}{l}\mathrm{CNN} / 5 / 6^{\text {th }}, 9^{\text {th }}, 13^{\text {th }} \\
/ 16\end{array}$ & Noun \\
\hline 37. Primary & $\begin{array}{l}\text { A preliminary election to appoint } \\
\text { delegates to a party conference or } \\
\text { to select the candidate for a } \\
\text { principal, especially presidential } \\
\text { election. }\end{array}$ & $\begin{array}{l}\mathrm{CNN} / 5 / 2^{\text {nd }}, 3^{\text {rd }}, 6^{\text {th }}, \\
8^{\text {th }}, 9^{\text {th }}, 10^{\text {th }}, 11^{\text {th }}, 13^{\text {th }}, \\
17^{\text {th }}, 18^{\text {th }}, 23^{\text {rd }}, 24^{\text {th }}, \\
30^{\text {th }} / 16\end{array}$ & $\begin{array}{l}\text { Adjectiv } \\
\text { e }\end{array}$ \\
\hline 38. Party & $\begin{array}{l}\text { A formally constituted political } \\
\text { group that contest election and } \\
\text { attempts to form or take part in } \\
\text { government. }\end{array}$ & $\begin{array}{l}\mathrm{CNN} / 5 / 2^{\text {nd }} / 16 \\
\mathrm{CNN} / 5 / 3^{\text {rd }}, 5^{\text {th }}, 6^{\text {th }}, \\
7^{\text {th }}, 9^{\text {th }}, 10^{\text {th }}, 11^{\text {th }}, 12^{\text {th }}, \\
13^{\text {th }}, 20^{\text {th }}, \\
26^{\text {th }} / 16\end{array}$ & Noun \\
\hline 39. Poll & $\begin{array}{l}\text { The process of voting in an } \\
\text { election. }\end{array}$ & $\begin{array}{l}\mathrm{CNN} / 5 / 3^{\text {rd }}, 5^{\text {th }}, 9^{\text {th }}, \\
10^{\text {th }}, 22^{\text {nd }}, 23^{\text {th }} / 16\end{array}$ & Noun \\
\hline
\end{tabular}




\begin{tabular}{|c|c|c|c|}
\hline 40. Policy & $\begin{array}{l}\text { A course or principle of action } \\
\text { adopted or proposed by a } \\
\text { government, party, business, or } \\
\text { individual. }\end{array}$ & $\begin{array}{l}\mathrm{CNN} / 5 / 3^{\text {rd }}, 6^{\text {th }}, 7^{\text {th }} \\
13^{\text {th }}, 14^{\text {th }}, 24^{\text {th }} / 16\end{array}$ & Noun \\
\hline 41. Political & $\begin{array}{l}\text { Relating to the government or the } \\
\text { public affairs of a country. }\end{array}$ & $\begin{array}{l}\mathrm{CNN} / 5 / 3^{\text {rd }}, 5^{\text {th }}, 6^{\text {th }} \\
9^{\text {th }}, 10^{\text {th }}, 12^{\text {th }}, 17^{\text {th }} \\
18^{\text {th }}, 24^{\text {th }}, 25^{\text {th }}, 27^{\text {th }} \\
/ 16\end{array}$ & $\begin{array}{l}\text { Adjectiv } \\
\text { e }\end{array}$ \\
\hline 42. Politic & $\begin{array}{l}\text { (of an action) seeming sensible } \\
\text { and judicious the circumstances. }\end{array}$ & $\begin{array}{l}\mathrm{CNN} / 5 / 8^{\text {th }}, \\
17^{\text {th }} / 16\end{array}$ & $\begin{array}{l}\text { Adjectiv } \\
\mathrm{e}\end{array}$ \\
\hline 43. $\quad$ Presidency & The office of president. & $\mathrm{CNN} / 5 / 6^{\text {th }} / 16$ & Noun \\
\hline 44. Radical & $\begin{array}{l}\text { Advocating or based on through } \\
\text { or complete political or social } \\
\text { change; representing or } \\
\text { supporting an extreme or } \\
\text { progressive section of political } \\
\text { party. }\end{array}$ & $\mathrm{CNN} / 5 / 5^{\text {th }} / 16$ & Noun \\
\hline 45. Republican & $\begin{array}{lr}\text { A person } & \text { advocating or } \\
\text { supporting } & \text { republican } \\
\text { government } & \end{array}$ & $\begin{array}{l}\mathrm{CNN} / 5 / 1^{\mathrm{st}}, 2^{\text {nd }}, 3^{\text {rd }}, \\
4^{\text {th }}, 5^{\text {th }}, 6^{\text {th }}, 7^{\text {th }}, 9^{\text {th }}, \\
11^{\text {th }}, 12^{\text {th }}, 14^{\text {th }}, 19^{\text {th }}, \\
24^{\text {th }}, 23^{\text {rd }}, 28^{\text {th }} / 16\end{array}$ & Noun \\
\hline 46. Right & $\begin{array}{l}\text { A grouping or political party } \\
\text { favoring conservative views and } \\
\text { supporting capitalist economic } \\
\text { principles. }\end{array}$ & $\begin{array}{l}\mathrm{CNN} / 5 / 5^{\text {th }} \text { and } 16^{\text {th }} \\
/ 16\end{array}$ & $\begin{array}{l}\text { Adjectiv } \\
\text { e }\end{array}$ \\
\hline 47. Senate & $\begin{array}{l}\text { Any of various legislative or } \\
\text { governing bodies, in particular. }\end{array}$ & $\begin{array}{l}\mathrm{CNN} / 5 / 3^{\mathrm{rd}}, 5^{\text {th }}, 13^{\text {th }} \\
22^{\text {nd }}, 28^{\text {th }} / 16\end{array}$ & Noun \\
\hline 48. Senator & A member of a senate. & $\begin{array}{l}\mathrm{CNN} / 5 / 3^{\mathrm{rd}}, 4^{\text {th }}, 6^{\text {th }} \\
7^{\text {th }}, 11^{\text {th }}, 28^{\text {th }} / 16\end{array}$ & Noun \\
\hline 49. Spokesman & $\begin{array}{l}\text { A person, especially a man, who } \\
\text { makes statement on behalf of } \\
\text { another individual or a group }\end{array}$ & $\begin{array}{l}\mathrm{CNN} / 5 / 5^{\text {th }} \\
17^{\mathrm{th}} / 16\end{array}$ & Noun \\
\hline 50. Strategy & $\begin{array}{l}\text { A plan of action or policy } \\
\text { designed to achieve a major or } \\
\text { overall aim. }\end{array}$ & $\begin{array}{l}\mathrm{CNN} / 5 / \\
23^{\mathrm{rd}} / 16\end{array}$ & Noun \\
\hline 51. Vote & $\begin{array}{l}\text { A formal indication of a choice } \\
\text { between two or more candidates } \\
\text { or courses of action, expressed } \\
\text { typically through a ballot or a } \\
\text { show of hands or by voice. }\end{array}$ & $\begin{array}{l}\mathrm{CNN} / 5 / \\
24^{\text {th }} / 16\end{array}$ & Verb \\
\hline 52. Voter & $\begin{array}{l}\text { A person who votes or has the } \\
\text { right to vote at an election. }\end{array}$ & $\begin{array}{l}\mathrm{CNN} / 5 / \\
24^{\text {th }} / 16 \\
\end{array}$ & Noun \\
\hline 53. Re-election & $\begin{array}{l}\text { The election of someone to a } \\
\text { further term of office. }\end{array}$ & $\begin{array}{l}\mathrm{CNN} / 5 / 3^{\text {rd }} \text { and } 5^{\text {th }} \\
/ 16\end{array}$ & noun \\
\hline
\end{tabular}


Based on the table of data above, it was identified that there were 53 features that belong to the word form of political jargon of 73 jargons data from political website. These forms cover $72.60 \%$ of all the findings 73 of the form of jargon in Political Website.

\section{Phrase Form}

According to Kridalaksana (2008:66), phrase is two or more words that work together as a unit. The following table presented the phrase form of jargon in Political Website:

Table 3 The Phrases Form of Jargon in Political Website

\begin{tabular}{|c|c|c|c|c|}
\hline No & $\begin{array}{l}\text { Phrases } \\
\text { Form }\end{array}$ & $\begin{array}{l}\text { Meaning of Jargon in } \\
\text { Political Website }\end{array}$ & $\begin{array}{c}\text { The title of } \\
\text { news pages in } \\
\text { CNN Politics }\end{array}$ & $\begin{array}{l}\text { Phrase } \\
\text { classes }\end{array}$ \\
\hline 1. & Fox news & TV conservative political cult & 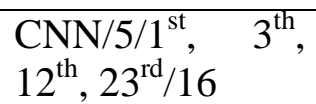 & Noun phrase \\
\hline 2. & $\begin{array}{l}\text { Republican } \\
\text { party }\end{array}$ & $\begin{array}{l}\text { One of the main us political } \\
\text { parties (the other being the } \\
\text { democratic party), favoring a } \\
\text { conservative stance, limited } \\
\text { central government and a } \\
\text { strong national defense. }\end{array}$ & $\begin{array}{l}\mathrm{CNN} / 5 / 1^{\text {st }}, 5^{\text {th }}, \\
11^{\text {th }}, 24^{\text {th }}, 25^{\text {th }} / 16\end{array}$ & Noun phrase \\
\hline 3. & $\begin{array}{l}\text { Golden } \\
\text { state }\end{array}$ & $\begin{array}{l}\text { A nickname for the state of } \\
\text { California. }\end{array}$ & $\mathrm{CNN} / 5 / 3^{\mathrm{rd}} / 16$ & Noun phrase \\
\hline 4. & $\begin{array}{l}\text { Public } \\
\text { relations }\end{array}$ & $\begin{array}{l}\text { The professional maintenance } \\
\text { of a favorable public image by } \\
\text { a company or other } \\
\text { organization or a famous } \\
\text { person }\end{array}$ & $\begin{array}{l}\mathrm{CNN} / 5 / 5^{\text {th }} \quad \text { and } \\
8^{\text {th }} / 16\end{array}$ & Noun phrase \\
\hline 5. & Blue collar & $\begin{array}{lll}\text { Relating to manual work } & \text { or } \\
\text { workers, } & \text { particularly } & \text { in } \\
\text { industry. } & & \end{array}$ & $\begin{array}{l}\mathrm{CNN} / 5 / 6^{\text {th }}, \quad 9^{\text {th }}, \\
24^{\text {th }} / 16\end{array}$ & $\begin{array}{l}\text { Adjective } \\
\text { phrase }\end{array}$ \\
\hline 6. & $\begin{array}{l}\text { White } \\
\text { house }\end{array}$ & $\begin{array}{l}\text { The official residence if the } \\
\text { US president in Washington, } \\
\text { DC. }\end{array}$ & $\begin{array}{l}\mathrm{CNN} / 5 / 5^{\text {th }}, \quad 9^{\text {th }}, \\
10^{\text {th }}, 13^{\text {th }}, 23^{\text {rd }}, \\
30^{\text {th }} / 16\end{array}$ & Noun phrase \\
\hline 7. & $\begin{array}{l}\text { Nominatio } \\
\mathrm{n} \\
\text { Conventio } \\
\mathrm{n}\end{array}$ & $\begin{array}{l}\text { A convention of a major } \\
\text { political party, especially one } \\
\text { that nominates a candidate for } \\
\text { presidency. }\end{array}$ & $\mathrm{CNN} / 5 / 22^{\text {nd }} / 16$ & Noun phrase \\
\hline 8. & Red state & $\begin{array}{l}\text { Traditionally a republican- } \\
\text { controlled state }\end{array}$ & $\mathrm{CNN} / 5 / 5^{\text {th }} / 16$ & Noun phrase \\
\hline
\end{tabular}




\begin{tabular}{|c|c|c|c|c|}
\hline 9. & Swing state & $\begin{array}{l}\text { A US state where the two } \\
\text { major political parties have } \\
\text { similar levels of support } \\
\text { among voters, viewed as } \\
\text { important in determining the } \\
\text { overall result of presidential } \\
\text { election. }\end{array}$ & $\begin{array}{l}\mathrm{CNN} / 5 / 5^{\text {th }} \text { and } \\
23^{\mathrm{rd}} / 16\end{array}$ & Noun phrase \\
\hline 10 & $\begin{array}{l}\text { Democrati } \\
\text { c party }\end{array}$ & $\begin{array}{l}\text { One of the main us political } \\
\text { parties (the other being the } \\
\text { republican party), which } \\
\text { follows a liberal program, } \\
\text { tending to promote a strong } \\
\text { central government and } \\
\text { expensive social group. }\end{array}$ & $\mathrm{CNN} / 5 / 23^{\mathrm{rd}} / 16$ & Noun phrase \\
\hline 11 & $\begin{array}{l}\text { Democrati } \\
\text { c socialist }\end{array}$ & $\begin{array}{l}\text { An advocate of democratic } \\
\text { socialism; a member of } \\
\text { political party whose are those } \\
\text { of democratic socialism. }\end{array}$ & $\begin{array}{l}\mathrm{CNN} / 5 / 12^{\mathrm{th}} / 16 \\
\mathrm{CNN} / 5 / 23^{\text {rd }} / 16\end{array}$ & $\begin{array}{l}\text { Adjective } \\
\text { phrase }\end{array}$ \\
\hline 12 & Ballot box & $\begin{array}{l}\text { A sealed box into which } \\
\text { voters put completed ballots. }\end{array}$ & $\mathrm{CNN} / 5 / 12^{\text {th }} / 16$ & Noun phrase \\
\hline 13 & $\begin{array}{l}\text { Battle } \\
\text { ground }\end{array}$ & $\begin{array}{l}\text { A military force created to } \\
\text { fight together, typically } \\
\text { consisting of several different } \\
\text { types of troops. }\end{array}$ & $\mathrm{CNN} / 5 / 24^{\text {th }} / 16$ & Noun phrase \\
\hline 14 & $\begin{array}{l}\text { Super } \\
\text { delegate }\end{array}$ & $\begin{array}{l}\text { An unelected delegated who is } \\
\text { free to support any candidate } \\
\text { for the presidential nomination } \\
\text { at the party's nation } \\
\text { convention. }\end{array}$ & $\begin{array}{l}\mathrm{CNN} / 5 / 1^{\text {st }} \text { and } \\
10^{\text {th }} / 16\end{array}$ & Noun phrase \\
\hline 15 & Super PAC & $\begin{array}{l}\text { A type of independent } \\
\text { political action committee } \\
\text { which may rise unlimited } \\
\text { sums of many from } \\
\text { corporations, unions, and } \\
\text { individuals but is not } \\
\text { permitted to contribute to or } \\
\text { coordinate directly with } \\
\text { parties or candidates. }\end{array}$ & $\mathrm{CNN} / 5 / 19^{\text {th }} / 16$ & Noun phrase \\
\hline
\end{tabular}

The next form of political jargon in Political Website is in the form of phrase. There were 15 features or $20.54 \%$ from the 73 of data political jargon the finding numbers in all the data. 


\section{Abbreviation Form}

According to Kridalaksana (2008:1), abbreviation is the result of shortened unit. The way of shortened takes the first syllable from the words. The following table presents the abbreviation form of jargon in Political Website.

Table 4 The Abbreviations Form of Jargon in Political Website

\begin{tabular}{|c|c|c|c|c|}
\hline No & $\begin{array}{l}\text { Abbreviation } \\
\text { s Form }\end{array}$ & $\begin{array}{l}\text { Complete } \\
\text { Words }\end{array}$ & $\begin{array}{l}\text { Meaning of Jargon in } \\
\text { Political Website }\end{array}$ & $\begin{array}{l}\text { The date of } \\
\text { news pages in } \\
\text { CNN Politics }\end{array}$ \\
\hline 1. & GOP & $\begin{array}{l}\text { Grand Old } \\
\text { Party }\end{array}$ & $\begin{array}{l}\text { The nickname of Republican } \\
\text { Party. }\end{array}$ & $\begin{array}{l}\mathrm{CNN} / 5 / 25^{\text {th }}, \\
26^{\text {th }}, 28^{\text {th }}, 19^{\text {th }}, \\
20^{\text {th }}, 22^{\text {nd }}, 24^{\text {th }}, \\
15^{\text {th }}, 13^{\text {th }}, 11^{\text {th }}, \\
16^{\text {th }}, 30^{\text {th }} / 16\end{array}$ \\
\hline $2 .$. & $\mathrm{RNC}$ & $\begin{array}{l}\text { Republican } \\
\text { Nation } \\
\text { Convention }\end{array}$ & $\begin{array}{l}\text { A convention of republican } \\
\text { party, especially one that } \\
\text { nominates a candidate for the } \\
\text { presidency. }\end{array}$ & $\begin{array}{l}\mathrm{CNN} / 5 / 11^{\text {th }}, \\
13^{\text {th }}, 19^{\text {th }}, 22^{\text {nd }}, \\
28^{\text {th }} / 16\end{array}$ \\
\hline 3. & VP & $\begin{array}{l}\text { Vice } \\
\text { President }\end{array}$ & $\begin{array}{l}\text { An official or executive } \\
\text { ranking below and deputizing } \\
\text { for a president. }\end{array}$ & $\mathrm{CNN} / 5 / 25^{\text {th }} / 16$ \\
\hline 4. & PAC & $\begin{array}{l}\text { Political } \\
\text { Action } \\
\text { Committee }\end{array}$ & $\begin{array}{l}\text { An organization that raises } \\
\text { money privately to influence } \\
\text { election or legislation, } \\
\text { especially at the federal level. }\end{array}$ & $\begin{array}{l}\mathrm{CNN} / 5 / 19^{\text {th }}, \\
27^{\text {th }}, 28^{\text {th }} / 16\end{array}$ \\
\hline 5. & NRA & $\begin{array}{l}\text { National } \\
\text { Rifle } \\
\text { Association }\end{array}$ & $\begin{array}{l}\text { A nation organization } \\
\text { founded in } 1871 \text { that } \\
\text { promotes the legal use of guns } \\
\text { and gun safety in the US and } \\
\text { defends a US citizen's } \\
\text { constitutional right to own } \\
\text { and bear arms. }\end{array}$ & $\mathrm{CNN} / 5 / 30^{\text {th }} / 16$ \\
\hline
\end{tabular}

The third form of political jargon in Political Website is in the form of abbreviation. There were $5(6.84 \%)$ of 105 political jargonthe finding numbers in all of the data.

\section{Acronym Form}

Acronym was the shortened a word or more become to only one word. The meaning of the word is the long of that word. Political website, There was no feature in acronym form from 73data of political jargons found in all of the data. 


\section{The Meaning of Jargon in Political Website}

According to Boer (1973:42-43), there are two kinds of word meanings based on the lexical meaning, they are denotative and connotative meanings. The writers confirmed the meanings of jargon by oxford dictionary and http://www.scholarastic.com/ teachers/article/ vocabulary -political-worlds.

\section{Denotative Meaning}

Denotative is the natural meaning and no addition. The meaning in the word not connected with the other thing, not explain in the connection with the other event. The denotative meaning is the meaning that suitable with the definition in the dictionary and it is limited. The following table presents the denotative meaning of jargon in Political Website.

Table 5 Denotative Meaning of Political Jargon in Political Website

\begin{tabular}{|c|c|}
\hline Jargon & Meaning \\
\hline 1. Accountability & The fact or condition of being accountable \\
\hline 2. Ballot & $\begin{array}{l}\text { A system of voting secretly and writing on a } \\
\text { particular issue / the piece of paper used to } \\
\text { record a person's vote. }\end{array}$ \\
\hline 3. Blowback & $\begin{array}{l}\text { The unintended adverse result of a political } \\
\text { action or situation. }\end{array}$ \\
\hline 4. Blowout & $\begin{array}{l}\text { An easy victory in a supporting contest or } \\
\text { election. }\end{array}$ \\
\hline 5. Budget & $\begin{array}{l}\text { Projected dollar amounts estimated ahead of } \\
\text { time for a project. }\end{array}$ \\
\hline 6. Bombshell & $\begin{array}{l}\text { An unexpected and surprising event, } \\
\text { especially an unpleasant one. }\end{array}$ \\
\hline 7. Caucus & $\begin{array}{l}\text { An information meeting of local party } \\
\text { members to discuss candidates and choose } \\
\text { delegates to the party convention. }\end{array}$ \\
\hline 8. Campaigner & $\begin{array}{l}\text { A person who work in an organized and active } \\
\text { way towards a goal. }\end{array}$ \\
\hline 9. Candidate & $\begin{array}{l}\text { A person who applies for a job nominated for } \\
\text { election. }\end{array}$ \\
\hline 10. Campaign & An organized effort to win an election \\
\hline 11. Coalition & $\begin{array}{l}\text { A temporary alliance for combined action, } \\
\text { especially of political parties forming a } \\
\text { government. }\end{array}$ \\
\hline 12. Democratic & $\begin{array}{l}\text { Relating or supporting democracy or its } \\
\text { principles }\end{array}$ \\
\hline 13. Democracy & $\begin{array}{l}\text { A system of government by the whole } \\
\text { population or all the eligible members of a } \\
\text { state, typically through elected representative }\end{array}$ \\
\hline
\end{tabular}




\begin{tabular}{|c|c|}
\hline 14. Democrat & A member of the Democratic Party. \\
\hline 15. Debate & $\begin{array}{l}\text { Held between the democratic nominees, } \\
\text { republican nominees, as well as in the } \\
\text { presidential campaign. }\end{array}$ \\
\hline 16. Delegate & $\begin{array}{l}\text { A person chosen at local level to represent the } \\
\text { state at the part's National Convention. }\end{array}$ \\
\hline 17. Declaration & $\begin{array}{l}\text { A formal or explicit statement or } \\
\text { announcement. }\end{array}$ \\
\hline 18. Election & $\begin{array}{l}\text { A formal and organized choice by vote of a } \\
\text { person for political officer or other position }\end{array}$ \\
\hline 19. Endorsement & $\begin{array}{l}\text { Come in three types: from celebrities, } \\
\text { newspaper, and political figures and } \\
\text { organization. }\end{array}$ \\
\hline 20. Ballot & $\begin{array}{l}\text { A system of voting secretly and writing on a } \\
\text { particular issue / the piece of paper used to } \\
\text { record a person's vote. }\end{array}$ \\
\hline 21. Blowback & $\begin{array}{l}\text { The unintended adverse result of a political } \\
\text { action or situation. }\end{array}$ \\
\hline 22. Endorse & Declare one's public approval or support of. \\
\hline 23. Escaping & Break free from confinement or control \\
\hline 24. Establishment & $\begin{array}{l}\text { A group in a society exercising power and } \\
\text { influence over matters of policy, opinion, or } \\
\text { taste and seen as resisting change. }\end{array}$ \\
\hline 25. Electorate & $\begin{array}{l}\text { All the people in a country or area who are } \\
\text { entitled to vote in an election. }\end{array}$ \\
\hline 26. Ideology & $\begin{array}{l}\text { A system of ideas and ideals, especially one } \\
\text { that forms the basic of economy or political } \\
\text { theory and policy }\end{array}$ \\
\hline 27. Leftist & $\begin{array}{l}\text { A person who supports the political views or } \\
\text { policies of left. }\end{array}$ \\
\hline 28. Liberal & $\begin{array}{l}\text { Willing to respect or accept behavior or } \\
\text { opinions different from one's own : open to } \\
\text { new idea. }\end{array}$ \\
\hline 29. Nomination & $\begin{array}{l}\text { The action of political party choose is official } \\
\text { candidate for a particular office. }\end{array}$ \\
\hline 30. Nominee & $\begin{array}{l}\text { The candidate chosen by a political party to } \\
\text { run for a particular office. }\end{array}$ \\
\hline 31. Opposition & $\begin{array}{l}\text { A group of opponents / resistance or dissent, } \\
\text { expressed in action or argument. }\end{array}$ \\
\hline 32. Opportunity & $\begin{array}{l}\text { A problem which does not have a clear } \\
\text { solution Someone's assignment }\end{array}$ \\
\hline 33. Primary & $\begin{array}{l}\text { A preliminary election to appoint delegates to } \\
\text { a party conference or to select the candidate } \\
\text { for a principal, especially presidential }\end{array}$ \\
\hline
\end{tabular}




\begin{tabular}{|c|c|}
\hline & election. \\
\hline 34. Party & $\begin{array}{l}\text { A formally constituted political group that } \\
\text { contest election and attempts to form or take } \\
\text { part in government. }\end{array}$ \\
\hline 35. Poll & The process of voting in an election. \\
\hline 36. Policy & $\begin{array}{l}\text { A course or principle of action adopted or } \\
\text { proposed by a government, party, business, or } \\
\text { individual. }\end{array}$ \\
\hline 37. Political & $\begin{array}{l}\text { Relating to the government or the public } \\
\text { affairs of a country. }\end{array}$ \\
\hline 38. Politic & $\begin{array}{l}\text { (of an action) seeming sensible and judicious } \\
\text { the circumstances. }\end{array}$ \\
\hline 39. Presidency & The office of president. \\
\hline 40. Radical & $\begin{array}{l}\text { Advocating or based on through or complete } \\
\text { political or social change; representing or } \\
\text { supporting an extreme or progressive section } \\
\text { of political party. }\end{array}$ \\
\hline 41. Republican & $\begin{array}{l}\text { A person advocating or supporting republican } \\
\text { government }\end{array}$ \\
\hline 42. Senate & $\begin{array}{l}\text { Any of various legislative or governing } \\
\text { bodies, in particular. }\end{array}$ \\
\hline 43. $\quad$ Senator & A member of a senate. \\
\hline 44. Spokesman & $\begin{array}{l}\text { A person, especially a man, who makes } \\
\text { statement on behalf of another individual or a } \\
\text { group }\end{array}$ \\
\hline 45. Strategy & $\begin{array}{l}\text { A plan of action or policy designed to achieve } \\
\text { a major or overall aim. }\end{array}$ \\
\hline 46. Vote & $\begin{array}{l}\text { A formal indication of a choice between two } \\
\text { or more candidates or courses of action, } \\
\text { expressed typically through a ballot or a show } \\
\text { of hands or by voice. }\end{array}$ \\
\hline 47. Voter & $\begin{array}{l}\text { A person who votes or has the right to vote at } \\
\text { an election. }\end{array}$ \\
\hline 48. Re-election & $\begin{array}{l}\text { The election of someone to a further term of } \\
\text { office. }\end{array}$ \\
\hline 49. Public relations & $\begin{array}{l}\text { The professional maintenance of a favorable } \\
\text { public image by a company or other } \\
\text { organization or a famous person }\end{array}$ \\
\hline $\begin{array}{ll}\text { 50. } & \text { Nomination } \\
\text { Convention }\end{array}$ & $\begin{array}{l}\text { A convention of a major political party, } \\
\text { especially one that nominates a candidate for } \\
\text { presidency. }\end{array}$ \\
\hline 52. Super delegate & $\begin{array}{l}\text { An unelected delegated who is free to support } \\
\text { any candidate for the presidential nomination } \\
\text { at the party's nation convention. }\end{array}$ \\
\hline
\end{tabular}




\begin{tabular}{|c|c|}
\hline 52. Super PAC & $\begin{array}{l}\text { A type of independent political action } \\
\text { committee which may rise unlimited sums of } \\
\text { many from corporations, unions, and } \\
\text { individuals but is not permitted to contribute } \\
\text { to or coordinate directly with parties or } \\
\text { candidates. }\end{array}$ \\
\hline 53. Ballot box & $\begin{array}{l}\text { A sealed box into which voters put completed } \\
\text { ballots. }\end{array}$ \\
\hline 54. GOP & The nickname of Republican Party. \\
\hline 55. $\mathrm{RNC}$ & $\begin{array}{l}\text { A convention of republican party, especially } \\
\text { one that nominates a candidate for the } \\
\text { presidency. }\end{array}$ \\
\hline 56. VP & $\begin{array}{l}\text { An official or executive ranking below and } \\
\text { deputizing for a president. }\end{array}$ \\
\hline 57. PAC & $\begin{array}{l}\text { An organization that raises money privately to } \\
\text { influence election or legislation, especially at } \\
\text { the federal level. }\end{array}$ \\
\hline 58. NRA & $\begin{array}{l}\text { A nation organization founded in } 1871 \text { that } \\
\text { promotes the legal use of guns and gun safety } \\
\text { in the US and defends a US citizen's } \\
\text { constitutional right to own and bear arms. }\end{array}$ \\
\hline
\end{tabular}

In the table above, it identified that there were 58 features that belong to the denotative meaning. These forms cover $79.45 \%$ of all the findings of the meaning of jargon in Political Website. Denotative meaning is simple meaning; this meaning indicates the references with no addition.

\section{Connotative Meaning}

The connotative meaning of jargon in political website is very important to know and understand what the speakers mean. Connotative meaning was the common meaning that has additional of certain feeling, emotion, value, and stimulus. In the other hand, connotative meaning is different with denotative meaning because there are some different interpretations between the people. It is based on the situation, condition and feeling. The following table presents the denotative meaning of jargon in Political Website.

Table 6 Connotative Meaning of Political Jargon in Political Website

\begin{tabular}{cll}
\hline No. & \multicolumn{1}{c}{ Jargon } & \multicolumn{1}{c}{ Meaning } \\
\hline 1. & Addressed & $\begin{array}{l}\text { To provide words on a topic which may have } \\
\text { little or no meaning }\end{array}$ \\
\hline 2. & Left & $\begin{array}{l}\text { Relating to a person or group favouring radical, } \\
\text { reforming, or socialist views. }\end{array}$
\end{tabular}




\begin{tabular}{|c|c|c|}
\hline 3. & Right & $\begin{array}{l}\text { A grouping or political party favoring } \\
\text { conservative views and supporting capitalist } \\
\text { economic principles. }\end{array}$ \\
\hline 4. & House & A legislative or declarative assembly \\
\hline 5. & Chairwoman & A female chairperson. \\
\hline 6. & Fox news & TV conservative political cult \\
\hline 7. & $\begin{array}{l}\text { Republican } \\
\text { party }\end{array}$ & $\begin{array}{l}\text { One of the main us political parties (the other } \\
\text { being the democratic party), favoring a } \\
\text { conservative stance, limited central } \\
\text { government and a strong national defense. }\end{array}$ \\
\hline 8. & Golden state & A nickname for the state of California. \\
\hline 9. & Blue collar & $\begin{array}{l}\text { Relating to manual work or workers, } \\
\text { particularly in industry. }\end{array}$ \\
\hline 10. & White house & $\begin{array}{l}\text { The official residence if the US president in } \\
\text { Washington, DC. }\end{array}$ \\
\hline 11. & Red state & Traditionally a republican-controlled state \\
\hline 12. & Swing state & $\begin{array}{l}\text { A US state where the two major political } \\
\text { parties have similar levels of support among } \\
\text { voters, viewed as important in determining the } \\
\text { overall result of presidential election. }\end{array}$ \\
\hline 13. & $\begin{array}{l}\text { Democratic } \\
\text { party }\end{array}$ & $\begin{array}{l}\text { One of the main us political parties ( the other } \\
\text { being the republican party), which follows a } \\
\text { liberal program, tending to promote a strong } \\
\text { central government and expensive social } \\
\text { group. }\end{array}$ \\
\hline 14. & $\begin{array}{l}\text { Democratic } \\
\text { socialist }\end{array}$ & $\begin{array}{l}\text { An advocate of democratic socialism; a } \\
\text { member of political party whose are those of } \\
\text { democratic socialism. }\end{array}$ \\
\hline & Battle ground & $\begin{array}{l}\text { A military force created to fight together, } \\
\text { typically consisting of several different types } \\
\text { of troops. }\end{array}$ \\
\hline
\end{tabular}

Not all the jargon in political website had denotative meaning; it also has connotative meaning although in the small frequency. Based on the table of data above there were $15(20.27 \%)$ of Connotative meanings of political jargon found in all of the data.

\section{The Function of Jargon in Political Website}

From the political website/ CNN Politics.com the writers found the function of jargon that writer founds that jargon in Political Website had three functions: 1) jargon can give a person a sense of belonging to a specific group of politics, 2) jargon can also make it easier for a person to communicate with their friends/ groups of politics, 3) jargon as effective signals for identification of politics. 


\section{DISCUSSION}

The use of language not only influenced by the linguistic factors but also it is influenced by the nonlinguistic factors. The nonlinguistic factors like social and situational factors. The social factors that influence in the use of language such as social status, educational level, age, gender, and the other. And then the situational factors like who the speaker is, what the language is, to whom, when, where, and what the problem is. According to Jendra (2010:19), the structure of language reflects the social function of language.

The language communities that exist is political. Political is the process of formation and distribution of power in society, among other tangible decisionmaking process, especially in countries, the activities of the government, politicians, or political parties, or the study of these activities.

Politics is one of the famous topics in the website for the society because in their life they are very close with the politics and government. So, it is important to know the language use in website politic which gave the all information about the political action in the word, several of website politics use the politics language or jargon politics for the website use. In this study, the political jargon focused on jargon used in American Legislative topic. The data sources were from news page in Political Website / CNN Politics.com.

Jargon had forms, meanings and functions in the usage. In this study, Political Website had different forms based on the word classes had different meaning and function.

\section{Jargon Forms}

Based on the classification of the word classes based on the process in morphology, there were jargon in word form, phrase form, abbreviation and acronym.

Table 7 The Percentage of Jargon Form in Political website

\begin{tabular}{|c|c|c|c|c|c|c|}
\hline \multirow[t]{2}{*}{ No. } & \multirow{2}{*}{ Finding } & \multicolumn{3}{|c|}{ Form of Jargon in Political } & \multirow{2}{*}{$\begin{array}{l}\text { Website } \\
\text { Acronym }\end{array}$} & \multirow{2}{*}{$\begin{array}{l}\text { Total } \\
\text { Data }\end{array}$} \\
\hline & & Word & Phrase & Abbr & & \\
\hline 1. & Frequency & 53 & 15 & 5 & 0 & 73 \\
\hline 2. & Percentage & $72.60 \%$ & $20.54 \%$ & $6.84 \%$ & $0 \%$ & $100 \%$ \\
\hline
\end{tabular}

From the table of data above, it can be seen that the forms of political jargon in word classifications there were 53(72.60\%) words, there were 15 (20.54\%) phrase, there were 5(6.84\%) abbreviation, and there was not acronym from $73(100 \%)$ the total data. The data showed that the highest forms of jargon were words forms with the persentages $72.60 \%$ and frequency were 53 words jargon. 
From the finding show there were $40(75.46 \%)$ noun, $9(16.98 \%)$ and 4 (7.54\%) verb classes of word forms from 53 of jargon forms in political website that writer found. In the phrase forms there were $13(86.66 \%)$ of noun phrases and $2(13.33 \%)$ of adjective phrases.

The forms of jargon in Political Website that can be found in this Study were in the form of word, phrase, abbreviation, and there was not acronym. The data were collected from internet, dictionary, book, article, etc.

\section{Jargon Meaning}

It is impossible that words have no meaning. Based on the data analyzed, the writer found that there were 58 jargons with denotative meaning and 15 jargons with connotative meaning. It show that in political website/ CNN Politics.com use jargon with the denotative meaning without a higher interpretation of meaning, and there were 15 jargon which need to be understood deeper or connotative. The frequency of occurrence of data can be seen in the table as follows:

Table 8 The Percentage of Meaning of Jargon in Political Website

\begin{tabular}{rllll}
\hline \multirow{2}{*}{ No. } & \multirow{2}{*}{ Finding } & \multicolumn{2}{c}{$\begin{array}{c}\text { Meaning of Jargon in Political } \\
\text { website. }\end{array}$} & \multirow{2}{*}{ Total data } \\
\cline { 3 - 4 } & & Denotative & Connotative & \\
\hline Frequency & 58 & 15 & 73 \\
\hline Percentage & $79.45 \%$ & $20.54 \%$ & $100 \%$ \\
\hline
\end{tabular}

From the table of data above, it can be seen that the difference of the frequency of the data was very high. The percentage of denotative meaning found in jargon in Political Website is higher than connotative meaning. There were 58 (79.45\%) denotative meanings of the total data and $15(20.54 \%)$ connotative meaning of 73 the total data.

\section{Jargon Function}

The third analyzed reveals the function of Jargon. It was a specialized term that had special function in communication. Each profession needs jargon. Based on Ives in Yuniasih (2013:18), Jargon has some function as a sense of belonging to a specific group, it easier for a person to communicate with their groups, and effective as signals for identification.

\section{Jargon can give a person a sense of belonging to a specific group}

The people who hear those words spoken by group of people, they can guess that they are the members of politics. The word 'Party' mean a formally 
constituted political group that contest election and attempts to form or take part in government. This word usually was using by political, the word 'Republican Party' means one of the main us political parties (the other being the democratic party), favoring a conservative stance, limited central government and a strong national defense. And the word of 'Democratic party' mean one of the main us political parties (the other being the republican party), which follows a liberal program, tending to promote a strong central government and expensive social group.

\section{Jargon can also make it easier for a person to communicate with their group}

The political website or CNN Politics used about jargon in their news like the words of 'Left' to said a person or group favoring radical, reforming, or socialist views. And word of 'Right' to said a grouping or political party favoring conservative views and supporting capitalist economic principles.

'Left' and 'Right' when communicate with people in the same group to make easier in communication. Users of political website were easier if they use jargon to communicate with. Because of that, jargon can be useful for communication between members and the groups.

\section{Jargon as effective signals for identification}

The people can understand what group, activity or occupation the other people belong to the conversation or read in the article. Jargon was a clear case of language being used for social identification. The word 'Campaign' used if an organized effort to win an election. The word a formal indication of a choice between two or more candidates or courses of action, expressed typically through a ballot or a show of hands or by voice. The words of 'vote' used for formal indication of a choice between two or more candidates or courses of action, expressed typically through a ballot or a show of hands or by voice. The abbreviation of 'GOP/Grand Old Party' is used for The nickname of Republican Party.

The readers that often used jargon in political website were easy to identify that they connected in politics. Jargon in political website made to use in activities that connected with the politics. The user of political website communities had their own language that was political website so it was easier to identify. Because of that jargon was the effective signals for identification in activities in this life.

Based on the explanation above, it can be concluded that all of jargon in Political Website/ CNN Politics had the function such as jargon can give a person a sense of belonging to a specific group, jargon can also make it easier for a person to communicate with their group and jargon as effective signals for identification. 


\section{CONCLUSION}

Jargon in Political Website had four forms like word, phrase, abbreviation and acronym. The forms of political jargon in word classification were 53 (72.60\%), phrase were $15(20.54 \%)$, abbreviation were $5(6.84 \%)$, and there was no acronym $(0 \%)$ from $73(100 \%)$ of total data.

There were two meanings of jargon in Political Website; they were denotative and connotative meanings. The percentage of denotative meaning found in jargon in Political Website was higher than connotative meaning. There were $58(79.72 \%)$ denotative meanings and 15 (20.27\%) connotative meaning of 73 the total data. Denotative meaning found of jargon in Political Website had the higher frequency than connotative meaning.

Jargon in Political Website had three functions such as jargon can give a person a sense of belonging to a specific group, jargon can also make it easier for a person to communicate with their members group and jargon as effective signals for identification.

\section{REFERENCES}

Boer, J. (1973). Basic Language. New York: Happer\&Row, Publisher,inc.

Bowo, E. (2014). Panel Panduan Untuk Web Master. Jakarta: Jasakom.

Drezner, D. W., \& Farrel, H. (2008). The power and politics of blogs. Public Choice, 134, 15-30. doi:10.1007/s11127-007-9198-1.

Hornby, A.S. (2001). Oxford Advance Learner's Dictionary. Oxford: Oxford University Press.

IPSOS.com. (2014).New Global Survey Rank CNN As Top International News brand. Retrieved From http://cnnpressroom.blogs.cnn.com/2014/10/16/new-global-survey-rankscnn-as-top-international-news-brand/

Jendra, M. I. I. (2010). Sociolinguistics the study of societies' language. Yogyakarta: GrahaIlmu.

Jogiyanto, HM. (2009). SistemTeknologiInformasi. Yogyakarta: Andi Yogyakarta.

Kridalaksana, H. (2008). Kamus Linguistik edisi keempat. Jakarta: PT Gramedia Pustaka Utama.

Satyakumara.com (2013).Top 15 Portal Berita online dunia, versi website ebizma.com.Retrieved from http://satyakumara.blogspot.co.id/2013/02/15-website-berita-terpopuler-di-dunia.html

Yuniasih, E. (2013). An Analysis of Jargon in Opera Van Java: English Education Study Program (S1 Thesis). Faculty of Teacher Training And Education Sarjana wiyata Tamansiswa University Yogyakarta, Yogyakarta 